International Journal of Engineering \&Technology, $7(3.12)(2018) 380-383$
International Journal of Engineering \& Technology
SPC
Website: www.sciencepubco.com/index.php/IJET
Research paper

\title{
Maximization of Lifetime of Wireless Sensor Network with Sensitive Power Dynamic Protocol
}

\author{
Manish Bhardwaj $^{* 1}$, Anil Ahlawat ${ }^{2}$, Nidhi Bansal ${ }^{3}$ \\ ${ }^{1,3}$ Computer Science and Engineering, SRM IST, India \\ ${ }^{2}$ Computer Science and Engineering, KIET, India \\ *Corresponding Author E-mail: ' aapkaapna13@gmail.com, 22 dranilahlawat@gmail.com, \\ 3nidhi18jul@gmail.com
}

\begin{abstract}
A vitality effective protocol configuration is a key testing issue in a network of Wireless Sensor. A portion of the few existing vitality effective protocols plots dependably forward the bundles through the base vitality based ideal course to the sink to limit vitality utilization. It causes a disturbed dispersion of remaining vitality between sensor nodes, which prompts partitioning of the network. The prime objective of this method is to pass the data packets to destination node through the vitality denser range within Sensor Networks Lifetime. The current procedure Energy Balanced Routing Protocol (EBRP) neglects to accomplish Throughput, Delay part, keeping in mind the end goal to enhance the Network Lifetime and Performance so the proficient steering convention is required with the abilities of both the Power Efficient and Power Balancing. To resolve this problem, this manuscript proposed Impediment Sensitive Power Unbiased Dynamic Routing Protocol (ISPUDRP). The proposed steering system accomplishes as far as End-to-End Delay, Throughput and Lifetime of network. This manuscript shows that proposed calculation accomplishes better execution performance than the current strategies.
\end{abstract}

Keywords: WSN, EBRP, ISPUDRP, Vitality, Lifetime of Network.

\section{Introduction}

WSNs are a progression of sensors unevenly or, on the other hand equally conveyed over an immense range used to screen hazardous situations, attack sensitive zones, timberland fires and so forth. The sensors are situated indiscriminately areas and handoff their data to a focal base that is for the most part a long way from the area of sensor node. Sensors normally have a couple of essential properties that join them: at least one sensor, a radio handset for correspondence, a microcontroller for calculation and basic leadership and a battery for vitality. The system ought to have the self-sorting out ability since the places of individual node are not known at first [1]. Collaboration among the node is the principle key point of this system. The gathering of node collaborates to disperse the accumulated data to other nodes in the system. The vital function regions of sensor systems are military ranges, cataclysmic event and wellbeing. Likewise, this system is utilized to screen light, temperature and stickiness or other ecological factors for common application [2].

The Remote Sensor Nodes are relied upon to play essential part later on age systems to detect the physical world. It is exceptionally outstanding that the vitality is the most basic asset of sensor network powered by battery. To expand the lifetime of this organizes as far as might be feasible, the vitality effectiveness moves toward becoming the most imperative parameter amid the Protocol outline. In request to accomplish and utilize the constrained vitality at sensor node adequately, the as of late proposed directing plans are endeavoring to locate the base vitality way to the sink, which is utilized to improve the vitality use at node [3].
From the writing overview, in any case, it is watched that to concentrate on the productivity of vitality while planning conventions for WSNs isn't adequate and furthermore recognized that the irregular vitality consumption that is drastically lessens the age of systems and abatements sensors scope proportion. Also, this irregularity of vitality utilization awkwardness is surely bothersome long haul quality also, potency of the sensor network. The sensor network itself devours the vitality equitably, at that point the availability between the source and destination could be kept up for a more extended time and hence the system segment may be put off. This wonderful corruption of the system nodes could be clearly given generous increases. What's more, thus, it ought to be discerning to influence a reasonable exchange to off between both the vitality proficiency and the adjusted vitality utilization.

\section{Related Work}

From our previous overview, it is noticed that various written works concentrate on vitality proficient directing conventions which point is to locate an ideal best way to limit vitality utilization either on neighborhood node or in the entire WSN. Some preexisted protocols suffering from vitality imbalance.

J. M. Kim et al.[4] this paper improves the LEACH protocol with the help of cluster head election method. In this paper fuzzy logic technique is used and with the help of this technique, network chooses the cluster head so that lifetime of the network gets increased.

Manish [5] this paper apply a wireless power transfer and Backpressure Technique to reduce the power problem of the 
network nodes. This paper shows the simulation results by using the energy conservation algorithm. This paper increased the significant lifetime of the network. Link utilization, route maintenance and reduce overhead is the main objective of this paper.

Manish et al.[6] this paper brief the cooperative communication in different nodes which communicate with same data at a single instant of time. This paper uses the optimum relay nodes selection technique in CC network and with the help of this technique, it reduces the power consumption of the network.

Sotiris Nikoletseas et al. [7] this paper focus on wireless charging of the portable devices for this it is work on two different types of protocols one perform wireless charging and evaluate the charging efficiency and second maintain the balances of the chargers. This paper evaluates the results in real time environment.

Benard Mumo Makaa et al.[8] this paper simply demonstrates the application of solid state tesla coils. According to the results, this paper shows that tesla coils are highly capable of generating high voltage, high-frequency waveforms. It also shows that tesla coils are designed for wireless power transfer.

Manish et al. [9] describe the concept of reduction of power consumption in communication process. This manuscript describes the RECAODV algorithm which minimizes the energy consumption in the network and increase the lifetime of network It also explains the concept of wireless power transfer between the nodes of the network.

Manish et al.[10] describe the Energy Conservation algorithm in this manuscript. With the help of energy conservation mechanisms this manuscript shows significant results. This is also used another technique to resolve the power problem of the ad hoc network with magnetic resonance concept.

Manish ei al.[11] shows the performance comparison between the AODV, DSR and DSDV protocols in grid environment. This manuscripts show that AODV perform better then DSR and DSDV mostly every aspect of comparison.

Manish et al.[12] shows the power consumption in the ad hoc network and the mobility impact on the energy consumption. With the help of magnetic resonance concept it also resolves the power problem of the ad hoc network nodes.

The manuscript is structured with sections. The Section 1 portrays with outline of WSN and vitality adjusting in WSN. Second Section manages the Literature survey/ related works. Segment 3 describes the existing problem. Segment 4 depicts the Proposed Algorithm Impediment Sensitive Power Unbiased Dynamic Routing Protocol (ISPUDRP). Section 5 depicts the result analysis. Section 6 gives the final conclusion.

\section{Existing Problem}

This Existing Routing Protocol (EBRP) is work with the concept of leftover vitality and vitality thickness. According to this existing protocol the approach is to forward packet to sink by means of the dense vitality zone which is expanding the lifetime of nodes. According to this plan a best vitality thickness based course amongst source and goal. But it doesn't work with shortest path and because of this the network performance demolish in terms of Throughput, Delay Factor and Network Lifetime. In this way this exploration paper planned an effective Impediment Sensitive Power Unbiased Dynamic Routing Protocol (ISPUDRP), which will enhance the system execution in terms of Throughput, Delay factor and Network Lifetime.

\section{Proposed Impediment Sensitive Power Unbiased DRP (ISPUDRP)}

In WSNs, the most important need is to bank the power of sensor nodes of the network. Previous research and the current research all are focused to reduce the power consumption and balanced the power of the nodes so that the network lifetime can be increase.

The architecture of smart delay aware shortest path and Impediment sensitive Power Unbiased Dynamic routing Protocol for WSN give the better opportunities for lesser power consumption and increased lifetime of the network. The proposed protocol enhances the performance of network in terms of find out the shortest energy density route so that for communication small amount of power will consume and that increase the lifetime of the network.

The proposed protocol of this manuscript execute in three phases:

1. Find the shortest route for destination in which each node have the unbiased power nature.

2. If the above point is affected by some reason then next alternate route must be available with same properties.

3 . After every change the route must be updated

\section{Smallest Route Based on Power}

This manuscript finds the shortest route in which each node has the unbiased power properties. The process of finding the route is given below.

Power_Density $=$ calculatePowerDensity () ;

$\theta d=\left(\right.$ L_Height $+\mathrm{I}+\mathrm{PDR} \_$size $) / \mathrm{u} \_$Msg.Height +1

Small $\bar{L}$ Lane(Ud $\theta \mathrm{d}>1$ ? $1-\mathrm{I} / \theta \mathrm{d}-1)$

Lin_Cap = Small_Lane $($ Route reply+Clock size $)$

$\theta$ ed $=u$ _Msg.Energy_Density/Local_Energy_Density

Small Lane $($ Ued $=\bar{\theta}$ ed $>1$ ? 1 - I $/ \bar{\theta}$ ed: $\theta$ ed $-\overline{1})$

$\theta \mathrm{e}=\mathrm{u} \_$Msg.Power $/$Power

Small_Lane(Ue日e $>1$ ? 1-I/ $/ \mathrm{e}-1)$

$\mathrm{Um}-(\overline{1-} \alpha-\beta) . \mathrm{Ud}+\alpha \mathrm{Ued}+\beta \mathrm{Ue}$

$\mathrm{C}=$ Dist (neighbor_ID)

$\mathrm{D}=\mathrm{Um} / \mathrm{C}$

Lin_Cap $=$ BW+Clock Size - route reply

RoutingTableUpdate(neighbor_ID)

Select the Lowest Height from the above table as LH

1. if (Local_Height $>$ LH +1 ) then

2. setLocal Height $(\mathrm{LH}+1)$

3. Endif

\section{Selection of Other Route}

This phase is the next phase of the planned protocol. In this next phase other route will be finding with the help of Other_Small_lane mechanisms. In this mechanisms a Other_small_lane() function is call and this function give the best shortest path. If the path power is reduced to lower the threshold level then some other alternate path will be follow. With the help of this step increase the sensor network reliability and it also increase the age of the network.

\section{Updating of Route}

In this phase after every step the route will be update with new route. This step is necessary because only finding of new route will increase the life of the network. This step increases the Throughput and reliability of the sensor network.

\section{Pseudo Code of Proposed Protocol}

CALL Small_Lane (calculatePowerDensity ())

Choose Finest Lane

FOR $t$ recorded data to be passing DO

BEGIN

IF Lane execution fine

CALL packet delivered

ELSE IF

CALL Other_Small_Lane (calculatePowerDensity())

Choose Other Finest Lane

END

\section{Results Analysis}

Execution of our proposed Algorithm is actualized with 
QualNet4.5 performance Simulator and ISPUDRP is altogether considered and assessed in this area as far as in terms of End-toEnd Delay, Throughput and Network Lifetime. According to the results found after simulation the proposed algorithm is perform better than existing Energy Balanced Routing Protocol (EBRP) in context of Throughput and End-to-End Delay and this proposed work holds all the positive highlights of Network Lifetime.

From the Figure 1, it is noticed that our proposed ISPUDRP finds the most limited way before as contrasted the EBRP. The proposed protocol gives the better result than the existing protocol because it works continuously for calculating the next alternate path and working with current calculating path and this property reduce the overhead of calculating the alternate path.

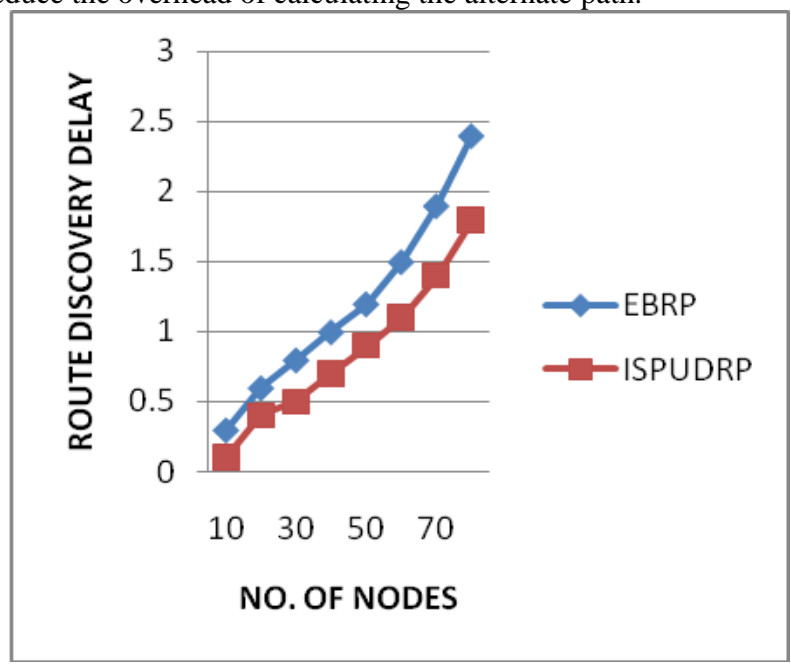

Fig. 1: Route Discovery Delay vs No. of Nodes

In this manuscript Figure 2 shows the graphical representation of End to End delay between ISPUDRP and EBRP protocols. The main Objective of the proposed protocol is to find the shortest path with good power density for communication between source and destination. With this property ISPUDRP takes less delay than EBRP protocol.

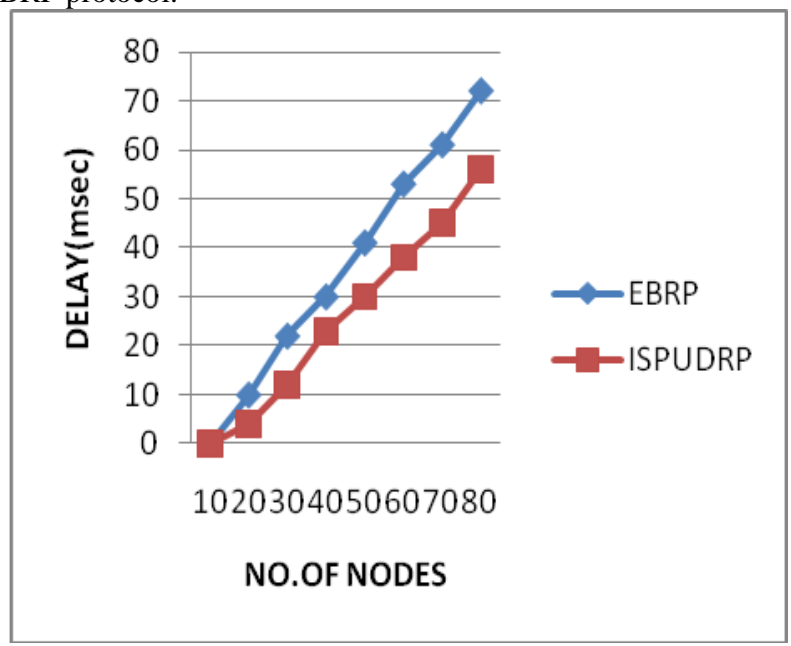

Fig. 2: Delay vs No. of Nodes

In this manuscript figure 3 shows the graphical representation of Throughput between ISPUDRP and EBRP protocols. According to the graph as the density of the nodes is less the proposed protocol gives the better results than EBRP protocol but as the number of sensor nodes increases the result of both protocol are almost same.

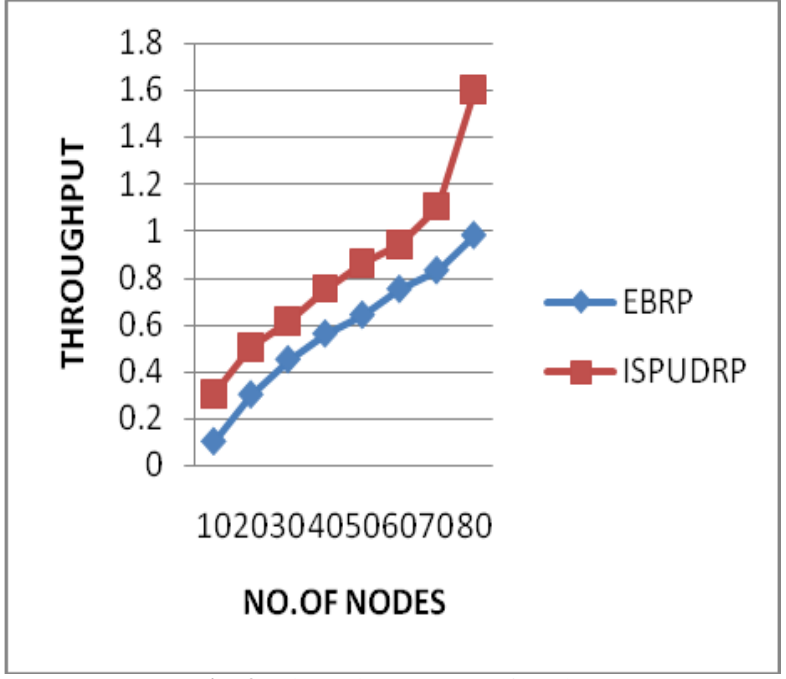

Fig. 3: Throughput vs No. of Nodes

Figure 4 of this manuscript shows the lifetime of the network in the form of line graphs. Proposed protocol pre calculate the next alternate path of the communication so that when power level of current path goes down to the threshold level than immediately communication continue with the alternate path so that the overhead and power consume in delay of the network is saved. This Increase the lifetime of the network.

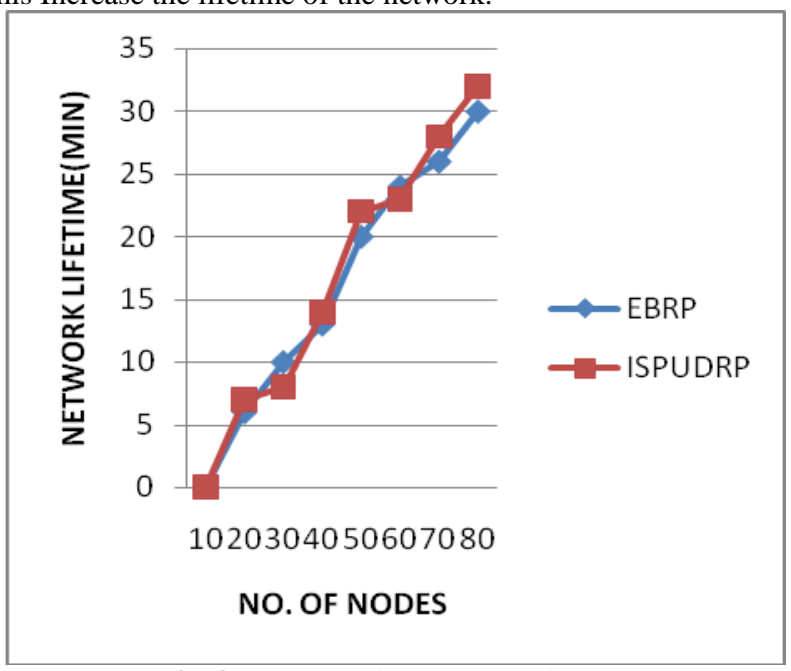

Fig. 4: Network Lifetime vs No. of Nodes

\section{Conclusion}

In Wireless Sensor Networks vitality is the serious issue because without the power any type of sensor network can't use in the communication process. This manuscript discusses the two power efficient routing protocols and their issues. In this manuscript we discuss several issues of both routing protocols and compare these two with reference of various performance metrics. Existing protocol fail to prove the performance in throughput and delay points so for this proposed protocol invoked. Comparison of these protocols is with the help of various performance metrics like delay, throughput, network lifetime and Route discovery delay. According to the experimental results the proposed protocol outperform than existing protocol in case of Throughput and end to end delay. Due to this performance the performance of the entire network will increase.

\section{References}

[1] Ren F., Zhang J., He T., Chuang Lin and Sajal K. Das (2011), EBRP: Energy-Balanced Routing Protocol for Data Gathering in 
Wireless Sensor Networks, IEEE Transactions On Parallel And Distributed Systems, Vol.22, No. 12.

[2] Liming H.(2007), Delay-Minimum Energy-Aware Routing Protocol (DERP) for Wireless Sensor Networks, IEEE Eighth ACIS International Conference on Software Engineering, Artificial Intelligence, Networking, and Parallel/Distributed Computing.

[3] Bhardwaj M., Ahlawat A. (2017), Reduce Energy Consumption in Ad hoc Network with Wireless Power Transfer Concept , International Journal of Control Theory and Applications, Vol. 10, Issue 13.

[4] Chung M.T., Han J.Y., Kim M. J. \& Park H.S. (2008). "CHEF: Cluster Head Election mechansim using Fuzzy logic in Wireless Sensor Networks", Proc. of ICACT, 654- 659.

[5] Bhardwaj M.(2015), Enhance life Time of Mobile Ad hoc Network using WiTriCity and Backpressure Technique, 1877-0509 (C) 2015 The Authors. Published by Elsevier B.V. This is an open access article under the CC BY-NC-ND license, doi: 10.1016/j.procs.2015.07.447

[6] Bhardwaj M.(2015), Selection of Efficient Relay for EnergyEfficient Cooperative Ad Hoc Networks. American Journal of Networks and Communications. Special Issue: Ad Hoc Networks. Vol. 4, No. 3-1, pp. 5-11. doi: 10.11648/j.ajnc.s.2015040301.12.

[7] Nikoletseas S. (2015), An experimental evaluation of wireless power transfer protocols in mobile ad hoc networks. IEEE Wireless Power Transfer Conference (WPTC) Page(s):1- 3.

[8] Makaa M. B. (2015), Analysis of WPT system using rearranged indirect-fed method for mobile applications, IEEE Wireless Power Transfer Conference (WPTC) Page(s):1 - 4. [3] X. Wu and G. Chen, and S.K. Das (2008), Avoiding Energy Holes in Wireless Sensor Networks with non uniform Node Distribution, vol. 19, no. 5, pp. 710-720.

[9] Bhardwaj M., Ahlawat A. (2017), Reduce Energy Consumption in Ad hoc Network with Wireless Power Transfer Concept International Journal of Control Theory and Applications, Vol. 10, Issue 13 .

[10] Bhardwaj M. \& Bansal A.(2015), Energy Conservation in Mobile Ad Hoc Network Using Energy Efficient Scheme and Magnetic Resonance. Advances in Networks. Special Issue: Secure Networks and Communications. Vol. 3, No. 3-1, pp. 34-39. doi:10.11648/j.net.s.2015030301.15.

[11] Bhardwaj M., Pathak A. \& Saraswat K. B. (2015), Optimum Experimental Results of AODV, DSDV \& DSR Routing Protocol in Grid Environment, Procedia computer science, 3rd International Conference on Recent Trends in Computing 2015 (ICRTC2015),Volume 57, Pages 1342-1350, doi:10.1016/j.procs.2015.07.451.

[12] Bhardwaj M. \& Singh A. (2015). Power Management of Ad Hoc Routing Protocols Using Mobility Impact and Magnetic Resonance. Advances in Networks. Special Issue: Secure Networks and Communications. Vol. 3, No. 3-1, pp. 27-33 doi:10.11648/j.net.s.2015030301.14.

[13] Han Z., Kim I. D., Lu X., Niyato D. \& Wang P. (2016), Wireless Charging Technologies: Fundamentals Standards and Network Applications, Communications Surveys \& Tutorials IEEE, vol. 18 pp. 1413-1452, ISSN 1553-877X.

[14] Nikoletseas S., Raptis P. T. \& Raptopoulos C. (2016), Interactive Wireless Charging for Energy Balance, IEEE 36th International Conference on Distributed Computing Systems (ICDCS), , pp. 262270, ISSN 1063-6927.

[15] Conti A., Francesco \& Passarella, (2009) Power conservation in wireless sensor networks: A survey, Ad Hoc Networks, vol. 7, No. 3 , pp. 537-568.

[16] Kumara, Lee O. K. \& Mitra (2009) Distributed Power Balanced Routing for Wireless Sensor Networks, Computer \& Industrial Engineering, vol. 57, issue 1, P.P. 125-135.

[17] Azzi D., Kanakaris V., Ndzi D. (2010), Ad-hoc networks energy consumption: A review of the ad hoc routing protocols, Journal of Engineering and Technology, review 3, pages 162-167.

[18] Huang H., Hu G. \& Yu F. (2010), A Routing Algorithm Based on Cross-layer Power Control in Wireless Ad Hoc Networks, Communications and Networking in China (CHINACOM).

[19] Ghanbarzadeh R. \& Meybodi R. M. (2010), Reducing message overhead of AODV routing protocol in urban area by using link availability prediction, Second International Conference on Computer Research and Development.

[20] Hussain A. M., \& Lovanjaneyulu N. (2011). IJCSIT International Journal of Computer Science and Information Technologies, Vol. 2 (3) , 1182-1186.
[21] Karadge S. P. \& Sankpal V. S. (2013) "A Performance Comparison of Energy Efficient AODV Protocols in Mobile Ad hoc Networks" International Journal of Advanced Research in Computer and Communication Engineering Vol. 2, Issue 1.

[22] Cousin B., Mekkakia Z., Smail O. (2011), "Energy efficiency in ad hoc wireless networks with node-disjoint path routing," 7 th Int. IEEE Workshop Systems Signal Processing and their Applications (WOSSPA), pp. 127-130.

[23] Kumar C., Patra N. A., Sharma K. d. (2013), “An Update based Energy-Efficient Reactive Routing Protocol for Mobile Ad Hoc Networks," International Journal Computer Network and Information Security, vol. 11, pp. 17-26. 